\title{
Data Dissemination Techniques in Vehicular Ad Hoc Network
}

\author{
Dharmendra Sutariya \\ Post graduate Student \\ Institute of technology, Nirma University, \\ Ahmedabad, India
}

\author{
Dr. S. N. Pradhan \\ Professor \\ Institute of technology, Nirma University, \\ Ahmedabad, India
}

\begin{abstract}
Numerous local incidents occur on road networks daily, many of which may lead to congestion and safety hazards. If vehicles can be provided with information about such incidents or traffic conditions in advance, the quality of driving can be improved significantly in terms of time, distance, and safety. Vehicular Ad Hoc Networks (VANETs) have newly emerged as an effective tool for improving road safety through the dissemination of warning messages among the vehicles in the network about potential obstacles on the road ahead. Various Approaches of data dissemination in vehicular network can be used to inform vehicles about dynamic road traffic condition so that a safe and efficient transportation system can be achieved. Here we extensively reviewed various data dissemination techniques and identify the challenges with it. However, type of VANET applications and inherent VANET characteristics such as different network density, fast movement of vehicles make data dissemination quite challenging.
\end{abstract}

\section{General Terms}

Vehicular Ad hoc Network (VANET), Data Dissemination, Flooding, Relaying.

\section{INTRODUCTION}

Many people lose their lives and/or are injured due to accidents or unexpected events taking place on road networks. Accidents and traffic jams generate a tremendous waste of time and fuel. If the vehicles are provided with timely and dynamic information related to road traffic conditions, any unexpected events or accidents, the safety and efficiency of the transportation system can be improved with respect to time, distance, fuel consumption. VANETs are ad hoc networks established among vehicles which are equipped with communication facilities. These vehicles are like a network nodes so that each node can act as the source of data, destination for data and a network router. Vehicular Ad Hoc Networks (VANET) is an effective tool for improving road safety through propagation of warning messages among the vehicles in the network about potential obstacles on the road ahead. In the envisioned applications, the equipped vehicles are able to communicate over the $5.9 \mathrm{GHz}$ frequency band via a Dedicated Short-Range Communication (DSRC) based device. DSRC with a range of up to $1000 \mathrm{~m}$ allows high-speed communications between vehicles for Intelligent Transport System (ITS) related applications [16]. These DSRC based applications for public safety and traffic management consist of intersection collision avoidance, warning messages, and approaching emergency vehicle warning etc. VANETs and Mobile Ad Hoc Networks (MANETs) have some similar characteristics such as short range of transmission, low bandwidth, omni-directional broadcast and limited storage capacity. They also obtained some different characteristics. We can define VANET characteristics as following.

1. High Dynamic topology: The speed of vehicles and choice of path defines the dynamic topology of VANET. If we assume two vehicles moving away from each other with a speed of $25 \mathrm{~m} / \mathrm{sec}$ and transmission range is about $250 \mathrm{~m}$, then the link between these two vehicles will available only 5 seconds. This defines its highly dynamic topology.

2. Frequent disconnected Network: Due to above feature the nodes needed another link with nearby vehicle to maintain seamless connectivity for longer communication. But in case of such failure, particularly in case of low vehicle density zone, frequent disruption of network connectivity will occur. Such problems can be deal with road-side deployment of relay nodes.

3. Mobility Modeling and Prediction: In volatile network topology connectivity between nodes requires knowledge of its positions and their movements which as such is very difficult to predict keeping in view the pattern of movement of each vehicle. A mobility model, node position prediction, predefined roadways model and vehicle speed are very important for effective network design.

4. Communication Environment: The mobility model highly varies from highways to that of city environment. The node prediction design and routing algorithm also be adapted for these changes. Highway mobility model is simple and predictable due to one-dimensional model. But for city mobility model, street structure, variable node density, presence of buildings and trees that behave as obstacles to even small distance communication make the model application that very complex and difficult.

5. Hard Delay Constraints: The safety aspect (such as accidents, brake event) of VANET application requires on time delivery of message to relevant nodes. It simply cannot compromise with any hard data delay in this regard.

6. Interaction with onboard sensors: This sensors helps in providing node location and their movement nature that are used for effective communication link and routing purposes.

VANET is a special application of MANET where data is propagated through exchange of messages between the vehicles. In VANETs, the restricted road topology imposes a directional nature to the message flow. Also due to higher node speeds and 
unstable connectivity among the nodes, it becomes essential that data be transmitted in the most efficient ways and with minimal delay. A VANET system architecture consists of different domains and many individual components as depicted in Figure 1. The figure shows three distinct domains (in-vehicle, ad hoc and infrastructure), and individual components (application unit, onboard unit, and road-side unit).

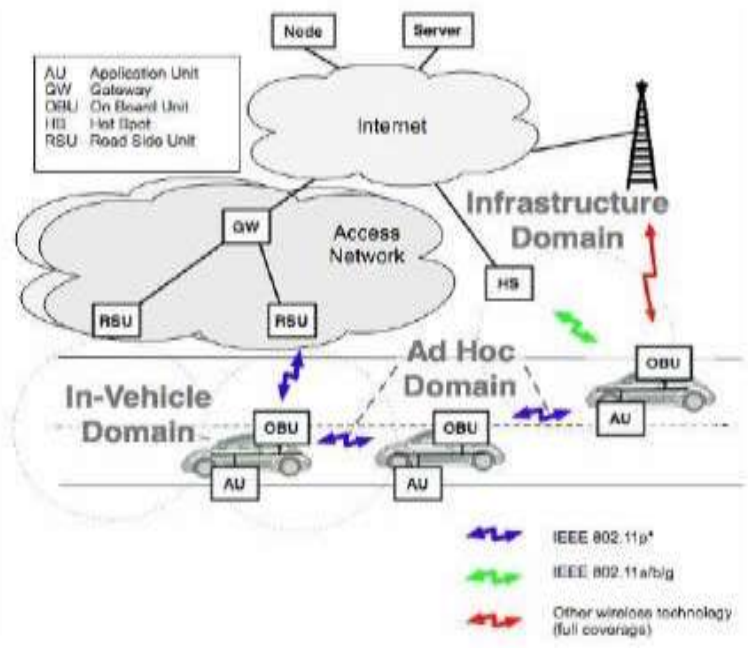

Figure 1. VANET Architecture [1]

Data dissemination among vehicles depends on the type of assumed network architecture. In the existence of infrastructures or road side units, two data dissemination approaches are assumed: push-based and pull-based. In the push-based approach, data is disseminated to anyone and suitable for popular data. In pull-based approach request-reply methodology is used and suitable for unpopular data propagation. With lacking of infrastructure two dissemination approaches can be considered: flooding and relaying. The flooding approach generally generates high message traffic. Therefore, the main challenge, encountered in this approach, is avoiding the broadcast storm problem [6]. With relaying approach there are also two challenges that may be encountered: 1) selecting relay points; and 2) ensuring reliability as selected nodes participate in packet retransmissions.

If the vehicles are provided with updated information regarding road traffic conditions, informed and intelligent decision help to take right actions to avoid being trapped in heavy traffic jams. Informed and intelligent decisions means a situation in which vehicles do not choose deliberately the congested paths due to unpredicted events and alternatively, can take available paths with less congestion level.

We organized this paper as follows. Section 2 describes V2I/I2V and V2V data dissemination approach. Section 3 describes the flooding based solution to suppress broadcast storm problem. Section 4 describes the relaying and associated challenges. Section 5 defines opportunistic flooding and section 6 concludes the article.

\section{DATA DISSEMINATION APPROACHES}

As defined in VANET architecture data dissemination between components is categorize as vehicle to infrastructure (V2I) or infrastructure to Vehicle (I2V) and vehicle to vehicle (V2V).

\subsection{Vehicle to Infrastructure (V2I)}

Push-based and Pull-based approaches are considered for V2I (or I2V) data dissemination.

In the push-based approach, the roadside unit broadcast the data to all vehicles which are in its range. Disadvantage is that everybody may not be interested to the same data. It is suitable for applications supporting local and public-interest data such as data related to unexpected events or accidents causing congestion and safety hazards. It also generates low contentions and collisions during packet propagation. In pull based approach vehicles are enabled to query information about specific targets and responses are routed toward them. It is useful for acquiring individualspecific data. It generates a lot of cross traffics including contentions and collisions during packet propagation.

Reference [2] is a research effort which considers information source (data center) to disseminate data to many vehicles on the roads. It is noted that periodically pouring data on the road is necessary since vehicles receiving the data may move away quickly, and vehicles coming later still need the data. Reference [3] is a research effort which focuses on the decentralized discovery of parking places. The proposed model consists of communication between vehicles and fixed infrastructures named as parking automat and also between vehicles.

\subsection{Vehicle to Vehicle (V2V)}

Flooding and relaying are two approaches that can be considered for vehicle to vehicle data dissemination.

In the flooding mechanism all types of data is broadcasted to neighbors. Whenever another vehicle receives a broadcast message, it stores and immediately forwards it by rebroadcast. It is suitable for delay sensitive applications and also for sparsely connected or fragmented networks. This mechanism is not scalable and generates broadcast storm problem due to high message overhead during rush hours or traffic jams. Therefore, there are several solutions proposed in order to avoid broadcast storm problem. It should be mentioned that these solutions are discussed in details in Section 3.

In the relaying mechanism relay node is selected for disseminating the messages. The relay node is responsible for forward the packet further. In this approach contention is less and it is scalable for dense networks. This is due to the less of number of the nodes participating in forwarding message and as a result generated overhead is less. However, selecting relay node and ensure reliability are two challenges that need to be addressed and are discussed in section 4 .

\section{BROADCAST STORM PROBLEM SUPPRESSION TECHNIQUES}

Flooding is the mechanism which can be used for data dissemination in vehicular ad hoc network which does not have any infrastructure support for communication. Due to shared wireless medium, flooding of data packets creates frequent contention and collisions among neighboring nodes. This problem is referred as broadcast storm problem. There are two main approaches that can deal with problem: 1) Simple forwarding restricted by the timer and number of hops; 2) Map based/geographic forwarding exploiting the map or geographic 
information such as directed flooding and aggregation. The details are described in subsections.

\subsection{Timer-Based (Simple Forwarding)}

A role-based multi-cast protocol is proposed in [4], [5] that Suppresses broadcast redundancy by assigning shorter waiting time prior to rebroadcast to more distant receivers. It should be noted that the primary objective of this study is to achieve Maximum reachability in a sparsely connected network. By knowing its own position, the system determines a waiting time WT depending on the distance $d$ to the sender such that the waiting time is shorter for more distant receivers as shown below:

$$
\begin{gathered}
W T(d)=-\frac{M a x W T}{\operatorname{Range}} * \hat{d}+\operatorname{Max} W T \\
\hat{d}=\operatorname{Min}\{d, \text { Range }\}
\end{gathered}
$$

Where MaxWT $=$ Maximum Waiting Time, Range $=$ transmission range and $\mathrm{d}=$ distance from sender

Three probabilistic and timer-based broadcast techniques are proposed by the authors in [6]. These techniques are used at network layer which are listed and described in the following:

- Weighted p-persistence (Vehicles located further have higher probability for retransmission)

- Slotted 1-persistence (Vehicles have to retransmit with probability 1 at the assigned time slot and shorter waiting time will be assigned to the vehicles locate further)

- $\quad$ Slotted p-persistence (Vehicles have to retransmit with probability $\mathrm{p}$ at the assigned time slot and shorter waiting time and higher probability will be assigned to the vehicles locate further)

Slotted 1-persistence and slotted p-persistence scheme can reduce broadcast redundancy and packet loss ratio up to 70 percent while still offering acceptable end to end delay for most multihop VANET applications.

\subsection{Hop Limited (Simple Forwarding)}

In hop limited approach we can avoid broadcast storm problem by simply limiting the number of hops the message gets propagated. However, determining the number of hops can be challenging.

Reference [7], is a research work that deploys strategy in which query disseminations are limited by the number of hops. The research discusses design issues relevant to a system for targeted ad delivery mechanisms for vehicles. AdTorrent searches for relevant ad-content using a hop-limited query broadcast. Since setting large hop-limit queries more nodes, a larger hop-limit improves the probability of finding the desired content and will likely increase the number of sources from which the content may be downloaded. There is an inherent trade-off between the reliability/effectiveness of the search and the flooding overhead. Thus, the hop limit in the query flood is a key design issue. An analytical model is presented in this research to estimate the performance impact of key design parameters such as the scope of the query flooding (determined by the number of hops) on the query hit ratio in epidemic query dissemination. This model can achieve 80 percent hit ratio with query hit limit value 4 .

\subsection{Directed Flooding (Map-based or Geographic Forwarding)}

Here flooding of data is restricted to specific directions or geographic areas. Therefore, by deploying this strategy, flooding of data in directions that does not benefit dissemination is limited.

In [8], Dissemination is defined based on propagation function for Inter-vehicular Networks. Propagation function encodes destination region and trajectory with using Map. They propose several flooding schemes as basic, probabilistic, function driven. Protocols defined below are designed to take decision in receiver based fashion so eliminate the need to collect neighbor information.

- $\quad$ One Zero Flooding (OZF) - Messages broadcasted only towards areas where propagation function returns the lower value.

- Distance Driven probabilistic diffusion (DDPD) Extension of OZF with addition of a probabilistic decision.

- Function Driven Probabilistic Diffusion (FDPD) - Use values return by function in order to calculate forwarding probability.

- $\quad$ Feedback Augmented Store, Forward Diffusion (FSFD) - Deploy store and forward techniques.

\subsection{Aggregation (Map-based or Geographic Forwarding)}

Aggregation is a technique proposed in some research works to avoid broadcast storm problem. Penetration and scalability are major challenges that can be faced in $\mathrm{V} 2 \mathrm{~V}$ based applications. With low market penetration rate, in the majority of the time there is no or only limited number of communication partners available within transmission range. Therefore, the average range in which information can be dispersed is small. Furthermore, Scalability becomes an issue once a higher market penetration is reached. In order to avoid overload conditions the amount of data transferred needs to be restricted.

To solve these two challenges, Segment-Oriented Data Abstraction and Dissemination (SODAD), a method for data dissemination for comfort applications, is proposed in [9]. Vehicle sensing data for on-board traffic information system and Data dissemination is reached by abstracting the map into adaptive segments and aggregation information (aggregation function) By restricting the method to the dissemination of map/position-based data, scalability is achieved. Here Adaptive broadcast intervals are defined based on provocation and mollification events to favor traffic conditions.

In Decentralized discovery of free parking places [3] Proactive dissemination scheme is used to inform drivers about parking place situation under urban traffic conditions. Periodic broadcast interval is used to disseminate received atomic and aggregated information which takes spatiotemporal character of parking places into account. Map is subdivided to overlay grids and information is classified into different levels for data dissemination. 


\section{RELAYING AND ASSOCIATED CHALLENGES}

Relaying is an approach for assigning the duty of forwarding a packet to specific nodes that satisfy some criteria. It generates less contention and scalable for dense network condition. The main challenges faced in the relay-based approaches include selecting the relay nodes and ensuring reliability. Basically, the relay-based data dissemination approaches can be divided into two categories: 1) simple forwarding and 2) map-based forwarding exploiting digital map information and GPS. With respect to the second challenge, ensuring reliability, several solutions such as RTS/CTS and ACK mechanisms are suggested. These techniques are described in following subsections.

\subsection{Relaying (Simple Forwarding)}

With respect to the research proposed in [10], a new efficient IEEE 802.11 based protocol, Urban Multi-hop Broadcast protocol (UMB), is proposed for VANET. The node farthest from the sender of the packet is selected as relay point. UMB is designed to address the broadcast storm, hidden node and reliability problems in multi-hop broadcast. It is composed of two phases: 1) directional broadcast and 2) intersection broadcast. In the first phase, sender nodes try to select the furthest node in the broadcast direction to assign the duty of forwarding and acknowledging the packet without any advance topology information i.e., sender selects the furthest node without knowing the ID or position of its neighbors. In the second phase, there are some repeaters installed at each intersection to disseminate the packets in all directions. When a node is selected to forward a packet which is out of repeater's transmission range it continues with the directional broadcast protocol. If the node is inside the transmission range of a repeater, it uses point-to-point IEEE 802.11 protocol. RTS/CTS like mechanism RTB/CTB is used between node and repeater to forward the data. ACK packet provides reliability of broadcast. After receiving this broadcast packet, the repeater uses directional broadcast in all road directions except the incoming packet direction.

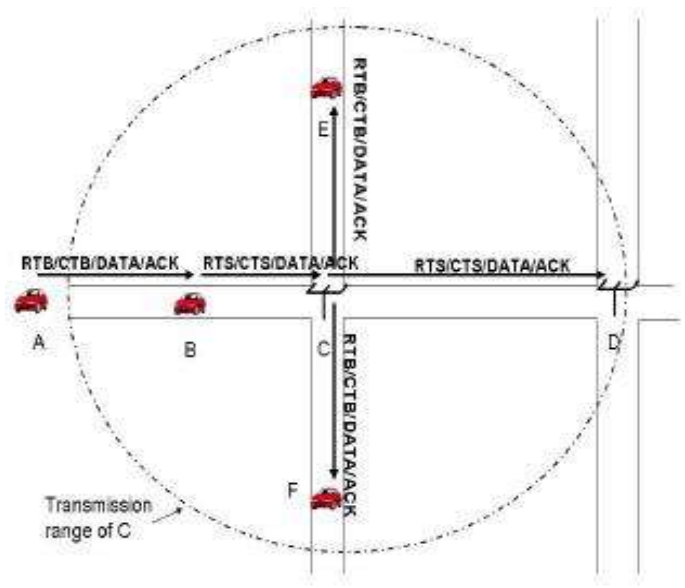

Figure 2. UMB Protocol [10]

An example of intersection handling is illustrated in Figure 2 which shows that vehicle A uses the directional broadcast to reach B. A is not in transmission range repeater C. Vehicle B is in the transmission range of $\mathrm{C}$ so $\mathrm{B}$ uses IEEE 802.11 protocol to communicate with repeater $\mathrm{C}$. Once repeater $\mathrm{C}$ receives the message, it initiates directional broadcasts. Repeater D is in the transmission range of repeater $\mathrm{C}$ so uses IEEE 802.11 protocol.

In [2] Data pouring and buffering dissemination scheme is proposed where disseminate data from center to many vehicles.

Each node maintain neighbor list and select farthest node as relay. Here dissemination zone defined as rectangle area and expiration time are attached as data delivery information. Ibers (Infostation) are deployed at intersections to broadcast data to the cross roads vehicles. They developed Analytical model for dissemination capacity and broadcast interval.

\subsection{Relaying (Map-based/ Geographic Forwarding)}

With respect to the research carried out in reference [11], the relaying is used. The relay point that forwards the message further is called "message head" which is defined as massage holder closest to the destination region. The proposed algorithm is called MDDV (Mobility-Centric Data Dissemination for Vehicular Networks). MDDV is designed to exploit vehicle mobility for data dissemination, and combines the idea of opportunistic forwarding, trajectory based forwarding and geographical forwarding. Opportunistic forwarding is used when the network is fragmented and end to end connectivity does not exist. Here messages are stored and forwarded as opportunities present themselves. Trajectory based forwarding directs messages along predefined trajectories. Generally, MDDV can be divided into two phases: forwarding phase and propagation phase. In the first phase, the data is forwarded to reach the destination region and in the second phase, the data is propagated to reach all the receivers in the region.

In research carried out in reference [12], Geographical routing is used to forward the query to the query region. Each node maintains a neighbor list and once query region is reached, nodes do flooding in that region. Reply is sent back to the source via flooding. All these tasks are done with use of four phases as dispatch-query, VAHS-computation, dispatch-reply and reply delivery.

\section{OPPORTUNISTIC FORWARDING}

Network fragmentation may happen due to the low market penetration rate or due to low traffic density periods. This data dissemination approaches are proposed where continuous network connectivity cannot be guaranteed.

With respect to the role-based multicast protocol proposed in [4], [5] the main objective is to achieve maximum reachability in a sparsely connected network. In the proposed approach, each node keeps a set named at neighboring set and retransmissions are based on changes in the neighbor sets.

With respect to the research carried out in reference [13], an opportunistic packet relaying is proposed for disconnected vehicular ad hoc network, named as OPERA. In OPERA, the packet progresses towards destination opportunistically, by a combination of data muling and local routing with the help of both co-directional and oncoming clusters. Cluster refers to the group of vehicles that are in a direct radio transmission with one another and have the same direction. In this research, some mathematical expressions are proposed such as the expected slot 
for a specific car, the probability of having disconnected periods and expected cluster size, both as a function of traffic density and communication range. Beacon message is sent for cluster formation and cluster maintenance. Head and tail vehicles in a cluster play an especial role in data dissemination.

In Fleanet [14] architecture is proposed for buy/sell queries dissemination on vehicular networks. Dissemination is basically by contacts, vehicles that receive queries store it in their database and see if there is a local match with it. Source node broadcasts queries periodically to its neighbors (opportunistic). Last encounter routing (LER) is used to send notifications from buyers to sellers.

In [15] shows the feasibility of information dissemination w.r.t. penetration ratio in city. With Analytical model they show that connectivity decreases with length. They propose installing SSUs (InfoStations), networked and stand-alone to improve dissemination by re-broadcasting the information. Vehicles periodically broadcast information to neighbors with use of Locomotion and Wireless propagation.

\section{CONCLUSION}

Push-based and pull-based are two approaches considered in V2I and $\mathrm{I} 2 \mathrm{~V}$. In the push-based approach, data is disseminated to anyone and it is suitable for specific data which is in the interest of anyone. In the pull-based approach, the network entities are able to query the required information and this approach is suitable for unspecific data propagation. In pull based approach generated cross traffic can cause interference and collisions among propagating data packets.

Flooding and relaying are the two proposed approaches for $\mathrm{V} 2 \mathrm{~V}$. In the flooding, reliable and quick data propagation is its advantages but not suitable for dense network condition due to its high message overhead. In the relaying approach, the duty of forwarding is assigned to less number of vehicles and generated overhead is less. Therefore, although it is suitable for dense networks, selecting the relay points and ensuring reliability are its disadvantages.

Opportunistic data dissemination approach proposed for sparsely connected network in which the data will be stored at the node until some nodes are available. This is the only applicable strategy in a sparsely connected network as there is no other option available.

Use of one or more data dissemination techniques depending on the type of application considered (either safety or comfort) and type of data to be transmitted among vehicles. However, the inherent VANET characteristic makes data dissemination quite challenging. As identifying the problem from above studies our future plan is to develop data dissemination technique which gives reasonable performance with different application and with variable VANET architecture.

\section{REFERENCES}

[1] Festag, G. Noecker, M. Strassberger, A. Lbke, B. Bochow, M. Torrent-Moreno, S. Schnaufer, R. Eigner, C. Catrinescu, and J. Kunisch," NoW - Network on Wheels: Project Objectives, technology and Achievements".In Proceedings of 6th international Workshop on Intelligent Transportation (WIT 2008), Hamburg, Germany, Mar 2008
[2] J. Zhao, Y. Zhang, G. Cao, "Data pouring and buffering on the road: A new data dissemination paradigm for Vehicular Ad Hoc Networks", IEEE Transactions on Vehicular Technology, vol. 56(6), pp: 3266-3277, 2007.

[3] M. Caliskan, D. Graupner, and M. Mauve ,'Decentralized discovery of free parking places", International conference on mobile computing and networking, in Proceedings of the 3rd International Workshop on Vehicular ad hoc network, 2006, pp. 30- 39.

[4] L. Briesemeister, L. Schafers, and G. Hommel, "Disseminating messages among highly mobile hosts based on Inter-vehicle communication", in Proceedings of the IEEE Intelligent Vehicles Symposium, 2000, pp. 522 -527.

[5] L. Briesemeister, and G. Hommel,"Role-based multicast in highly mobile but sparsely connected ad hoc networks", First annul workshop on Mobile and Ad Hoc Networking and Computing (MobiHOC) .2000, pp. 45-50.

[6] N. Wisitpongphan, O. K. Tonguz, J. S. Parikh, P. Mudalige, F. Bai, and V. Sadekar, "Broadcast storm mitigation techniques in Vehicular Ad hoc Networks", IEEE Wireless Communications, vol. 14 (6), pp. 84-94, 2007.

[7] A. Nandan, S. Tewari, S. Das, and L. Kleinrock,"'Modeling epidemic query dissemination in Adtorrent Network", in proceedings of IEEE CCNC, 2006, pp. 1173-1177.

[8] P. Costa, D. Frey, M. Migliavacca, and L. Mottola, "Towards lightweight information dissemination in Inter-vehicular Networks", International conference on mobile computing and networking, in Proceedings of the 3rd International workshop on Vehicular ad hoc networks, 2006, pp. 20- 29.

[9] L. Wischhof, A. Ebner, H. Rohling, "Information dissemination in selforganizing inter-vehicle networks, IEEE Transactions on intelligent Transportation Systems, vol. 6 (1), pp. 90- 101, 2005.

[10] G. Korkmaz, E. Ekici, F. zgner, and zgner, "Urban multihop broadcast protocol for Inter-vehicle communication systems", in Proceedings of the 1st ACM International Workshop on Vehicular Ad hoc Networks ,2004, pp. 76- 85.

[11] H. Wu, R. Fujimoto, R. Guensler, and M. Hunter,'MDDV: A mobility centric data dissemination algorithm for Vehicular Networks", in Proceedings of the 1st ACM International Workshop on Vehicular Ad hoc Networks, 2004, pp. 47-56.

[12] M. Dikaiakos, S. Iqbal, T. Nadeem, L. Iftode,"VITP: An Information Transfer Protocol for Vehicular Computing", VANET05, September 2, 2005, Cologne, Germany.

[13] M. Abuelela, S. Olariu, and I. Stojmenovic,"OPERA: Opportunistic Packet Relaying in Disconnected Vehicular Ad Hoc Networks", 5th IEEE International Conference Mobile Ad Hoc and Sensor Systems, 2008, pp. 285-294.

[14] U. Lee, J.-S. Park, E. Amir, and M. Gerla,'Fleanet: A virtual market place on vehicular networks" Proc. of V2VCOM, pages 18, July 2006.

[15] C. Lochert, B. Scheuermann, M. Caliskan, and M. Mauve, "The feasibility of information dissemination in vehicular adhoc networks", In Proc. of WONS, Austria, Jan. 2007.

[16] http://www.standards.its.dot.gov. 